\title{
PERFIL DOS PACIENTES COM TUBERCULOSE E OS FATORES ASSOCIADOS AO ABANDONO DO TRATAMENTO
}

Suellen Karina de Oliveira Giroti ${ }^{1}$, Renata Aparecida Belei ${ }^{2}$, Fernanda Novaes Moreno ${ }^{3}$, Fernando Salomão da Silva ${ }^{4}$

\begin{abstract}
RESUMO: O objetivo desta pesquisa foi identificar os fatores de risco associados ao abandono pelos pacientes do tratamento para tuberculose. Analisou-se 147 fichas de notificação de tuberculose do Sistema de Informações de Agravos de Notificação, do Município de Londrina, Paraná, em 2006. Verificou-se que 68\% eram pacientes do sexo masculino, 35,4\% tinham entre 41 e 60 anos, 22,4\% estudaram por 4 a 7 anos, e 42,8\% moravam em região de baixo nível socioeconômico; destaca-se que, dos 147 pacientes, $68 \%$ finalizaram o tratamento e $10,2 \%$ o abandonaram. Entre os abandonos, $80 \%$ eram do sexo masculino, $46,7 \%$ tinham entre $21 \mathrm{e} 40$ anos e 33,3\% estudaram de $4 \mathrm{a} 7$ anos. Conhecer o perfil dos pacientes que abandonam o tratamento para tuberculose é importante para subsidiar medidas de apoio. Urge melhorar a busca ativa daqueles que apresentam riscos para o abandono do tratamento, evitando a disseminação da doença e o aumento da morbimortalidade.
\end{abstract}

PALAVRAS-CHAVE: Tuberculose; Pacientes desistentes do tratamento; Fatores de risco.

\section{PROFILE OF PATIENTS WITH TUBERCULOSIS AND FACTORS RELATED TO THE TREATMENT ABANDONMENT}

\begin{abstract}
The purpose of this research was to identify risk factors associated with noncompliance of the patients treated for tuberculosis. We analyzed 147 records of tuberculosis notification from the Information System for Notifiable Diseases in the city of Londrina, Paraná, in 2006. It was found that $68 \%$ were male patients, $35.4 \%$ were between 41 and 60 years, $22.4 \%$ studied for $4-7$ years, and $42.8 \%$ lived in a region of low socioeconomic status; it stands that of the 147 patients, $68 \%$ have finished treatment and $10.2 \%$ have abandoned it. Among dropouts, $80 \%$ were male, $46.7 \%$ were between 21 and 40 years and 33.3\% had 4-7 years. Knowing the profile of patients who abandon treatment for tuberculosis is important to support relief. It is urgent to improve the active pursuit of those who pose a risk of treatment abandonment, avoiding the spread of the disease and mortality increasing.
\end{abstract}

KEYWORDS: Tuberculosis; Patient dropouts from treatment; Risk factors.

\section{PERFIL DE LOS PACIENTES CON TUBERCULOSIS Y LOS FACTORES ASOCIADOS AL ABANDONO DEL TRATAMIENTO}

RESUMEN: El objetivo de esta investigación fue identificar los factores de riesgo asociados al abandono por los pacientes del tratamiento para tuberculosis. Fueron analizadas 147 fichas de notificación de tuberculosis del Sistema de Informaciones de Agravios de Notificación, del Municipio de Londrina, Paraná, en 2006. Se verificó que 68\% eran pacientes del sexo masculino, 35,4\% tenían entre 41 y 60 años, 22,4\% estudiaron por 4 a 7 años, y 42,8\% vivían en región de bajo nivel socioeconómico; se destaca que, de los 147 pacientes, 68\% finalizaron el tratamiento y 10,2\% lo abandonaron. Entre los abandonos, 80\% eran del sexo masculino, 46,7\% tenían entre 21 y 40 años y 33,3\% estudiaron de 4 a 7 años. Conocer el perfil de los pacientes que abandonan el tratamiento para tuberculosis es importante para subsidiar medidas de apoyo. Urge mejorar la búsqueda activa de aquellos que presentan riesgos para el abandono del tratamiento, evitando la diseminación de la enfermedad y el aumento de la morbimortalidad.

PALABRAS CLAVE: Tuberculosis; Pacientes desistentes del tratamiento; Factores de riesgo.

${ }^{1}$ Enfermeira. Especialista em Controle de Infecção em Serviços de Saúde. Universidade Estadual de Londrina-UEL.

${ }^{2}$ Enfermeira. Doutora em Educação. Docente do Curso de Especialização em Controle de Infecção em Serviços de Saúde da UEL.

${ }^{3}$ Enfermeira. Especialista em Gerência dos Serviços de Enfermagem. UEL.

${ }^{4}$ Enfermeiro do Hospital Regional Vale do Ribeira, SP. Especialista em Enfermagem Médico Cirúrgica e Assistência Multiprofissional em Oncologia.

Autor correspondente:

Suellen Karina de Oliveira Giroti

Universidade Estadual de Londrina

Rua Alexander Graham Bell, 679 - 86063-250 - Londrina-PR

Recebido: 11/09/09

E-mail: suellenkarina@hotmail.com

Aprovado: 24/02/10 


\section{INTRODUÇÃO}

A tuberculose (TB) é uma doença de amplitude mundial e seu principal agente etiológico é o Mycobacterium tuberculosis, identificado em 1882 por Robert Koch. Esta enfermidade pode resultar da reativação de uma primo-infecção ou pode ser decorrente de infecção recentemente adquirida ${ }^{(1)}$.

Denomina-se caso de TB todo indivíduo com diagnóstico confirmado por baciloscopia ou cultura, e também aquele com diagnóstico médico baseado em dados clínico-epidemiológicos e resultados de exames complementares $^{(2)}$.

Estimativas indicam que, em 2006, 9,2 milhões de pessoas no mundo foram infectadas com o Mycobacterium tuberculosis, entre esses, 4,1 milhões eram de novos casos bacilíferos. Os países com maiores concentrações da doença são Índia, China, Indonésia, África do Sul e Nigéria( ${ }^{(3)}$. A transmissão se perpetua por contatos, principalmente dentro do lar, em locais de aglomeração de pessoas e no trabalho ${ }^{(4-5)}$.

No Brasil, no combate à $\mathrm{TB}$, um dos aspectos mais desafiadores é o abandono do tratamento, pois repercute nos índices de mortalidade e incidência da doença, e na multirresistência ao tratamento. Considerase abandono quando, após iniciado o tratamento, o indivíduo deixa de comparecer à unidade de saúde por mais de 30 dias consecutivos após a data aprazada para o retorno ${ }^{(4)}$.

Estima-se que em Londrina, município localizado na região norte do Estado do Paraná, cerca de 90 mil pessoas residam em microáreas críticas, onde a probabilidade de adoecimento por doenças infectocontagiosas e mortes prematuras é consideravelmente maior devido ao risco social. Com uma população de cerca de 470 mil habitantes, apresenta profundos contrastes sociais. Para ir ao encontro do que preconiza o Programa Nacional de Controle de Tuberculose, os gestores implementaram ações com atendimentos centralizados no Centro de Referência, porém, em 2002 iniciaram o processo de descentralização dos casos para as Unidades Básicas de Saúde, com a justificativa de agilização e qualificação das ações ${ }^{(2)}$.

Este estudo teve por objetivo identificar os fatores de risco relacionados ao abandono do tratamento para tuberculose no Município de Londrina, Estado do Paraná.

\section{METODOLOGIA}

O presente estudo é de natureza quantitativa, com base nos registros apresentados pela Vigilância Epidemiológica do Município de Londrina no Sistema de Informações de Agravos de Notificação (SINAN), após autorização formal da chefia deste serviço.

Foram incluídas inicialmente 152 fichas de agravos de notificação obrigatória de Tuberculose, emitidas de janeiro e dezembro de 2006 ao SINAN. Cada uma representa um novo tratamento, sendo que após 30 dias de abandono, se houver retorno do paciente, este é cadastrado como "reingresso" e é emitida uma nova ficha epidemiológica. Do total das fichas investigadas, foram observados cinco pacientes como "reingressos" após abandono, totalizando 147 pacientes no estudo.

Assim, fizeram parte do estudo os pacientes com tuberculose que possuíam ficha de notificação de tuberculose, modelo SINAN, preenchida no ano de 2006. O critério de exclusão foi ser um caso com registro de reingresso após abandono.

Os dados coletados das fichas de notificação foram sexo, cor, idade, escolaridade, tipo de entrada, forma de TB, agravos associados, HIV, resolução do caso e região de moradia. Estes foram sistematizados em tabela, analisados, agrupados em categorias temáticas e discutidos com base na literatura.

Foram respeitados os princípios éticos relacionados à pesquisa com seres humanos e o projeto foi aprovado pelo Comitê de Ética e Pesquisa da Universidade Estadual de Londrina sob parecer n. 225/08.

\section{RESULTADOS E DISCUSSÃO}

Entre os 147 pacientes investigados, 68\% eram do sexo masculino e $32 \%$ do sexo feminino, sendo a maioria de cor branca 53\%. Quando analisado o nível de escolaridade, percebeu-se que do total de pacientes estudados, 22,4\% tinham entre quatro e sete anos de estudo, sendo este tempo também observado entre os que abandonaram o tratamento 33,3\%. Diferentemente, uma pesquisa realizada em Bagé, Rio Grande do Sul, apontou que a maioria dos casos acometidos possuíam apenas de um a três anos de estudo, mas a idade variava entre 26 a $35 \operatorname{anos}^{(6)}$. Dessa forma, percebeu-se que, apesar da diferença de anos de estudo, a idade relacionada ao adulto jovem foi um fator de risco presente nos dois estudos (Tabela 1).

Os fatores de ordem sociocultural podem dificultar o tratamento, como o baixo nível de escolaridade, desconhecimento sobre a tuberculose, a nãoaceitação da doença e o fato de considerar-se curado antes da cura efetiva, já que os pacientes se sentem

Cogitare Enferm. 2010 Abr/Jun; 15(2):271-7 
melhor nos primeiros meses de tratamento ${ }^{(7)}$. Por isto, necessidade de atenção especial dos profissionais da quanto menor o tempo de escolaridade, maior será a saúde ao paciente em tratamento.

Tabela 1 - Perfil sociodemográfico e clínico dos pacientes com tuberculose. Londrina, 2006.

\begin{tabular}{|c|c|c|c|c|c|}
\hline \multirow[t]{2}{*}{ Variáveis } & & \multicolumn{2}{|c|}{ Casos notificados } & \multicolumn{2}{|c|}{$\begin{array}{c}\text { Casos de abandono de } \\
\text { tratamento }\end{array}$} \\
\hline & & $\mathbf{N}$ & $\%$ & $\mathbf{N}$ & $\%$ \\
\hline \multirow[t]{2}{*}{ Sexo } & Masculino & 100 & 68,0 & 12 & 80,0 \\
\hline & Feminino & 47 & 32,9 & 03 & 20,0 \\
\hline \multirow[t]{6}{*}{ Cor } & Branco & 78 & 53,0 & 05 & 33,3 \\
\hline & Negro & 17 & 11,6 & 02 & 13,3 \\
\hline & Pardo & 25 & 17,0 & 05 & 33,3 \\
\hline & Amarelo & 05 & 3,40 & 01 & 06,7 \\
\hline & Indígena & 01 & 0,70 & - & - \\
\hline & Não preenchidas & 21 & 14,3 & 02 & 13,3 \\
\hline \multirow[t]{6}{*}{ Idade } & $0-20$ & 18 & 12,3 & 02 & 13,3 \\
\hline & $21-40$ & 48 & 32,7 & 07 & 46,7 \\
\hline & $41-60$ & 52 & 35,4 & 05 & 33,3 \\
\hline & $61-80$ & 23 & 15,6 & 01 & 06,7 \\
\hline & 80 ou mais & 03 & 2,00 & - & - \\
\hline & Não preenchidas & 03 & 2,00 & - & - \\
\hline \multirow[t]{7}{*}{ Escolaridade } & $1-3$ & 18 & 12,3 & 03 & 20,0 \\
\hline & $4-7$ & 33 & 22,4 & 05 & 33,3 \\
\hline & $8-11$ & 26 & 17,6 & 01 & 06,7 \\
\hline & 12 ou mais & 13 & 8,90 & 01 & 06,7 \\
\hline & Ignorado & 19 & 13,0 & 03 & 20,0 \\
\hline & Nenhuma & 15 & 10,2 & 01 & 06,7 \\
\hline & Não preenchidas & 23 & 15,6 & 01 & 06,7 \\
\hline \multirow[t]{3}{*}{ Tipo de entrada } & Caso novo & 140 & 95,3 & 13 & 86,7 \\
\hline & Recidiva & 04 & 2,70 & 01 & 06,7 \\
\hline & Transferência & 03 & 2,00 & 01 & 06,7 \\
\hline \multirow[t]{3}{*}{ Forma de Tuberculose } & Pulmonar & 102 & 69,4 & 10 & 66,7 \\
\hline & Extra pulmonar & 36 & 24,5 & 05 & 33,3 \\
\hline & Ambas & 09 & 6,10 & - & - \\
\hline \multirow[t]{5}{*}{ Agravos associados } & Álcool & 20 & 13,6 & 02 & 13,3 \\
\hline & Aids & 10 & 6,80 & 01 & 06,7 \\
\hline & Drogas & 03 & 2,00 & - & - \\
\hline & Diabetes & 13 & 8,90 & - & - \\
\hline & Doença mental & - & - & 01 & 06,7 \\
\hline
\end{tabular}




\begin{tabular}{|c|c|c|c|c|c|}
\hline \multirow[t]{2}{*}{ Variáveis } & & \multicolumn{2}{|c|}{ Casos notificados } & \multicolumn{2}{|c|}{$\begin{array}{c}\text { Casos de abandono de } \\
\text { tratamento }\end{array}$} \\
\hline & & $\mathbf{N}$ & $\%$ & $\mathbf{N}$ & $\%$ \\
\hline \multirow[t]{3}{*}{ Agravos associados } & Outros & 09 & 6,10 & - & - \\
\hline & Ignorado & 80 & 54,4 & 08 & 53,3 \\
\hline & Não preenchidas & 12 & 8,20 & 03 & 20,0 \\
\hline \multirow[t]{5}{*}{ HIV } & Positivo & 10 & 6,80 & 01 & 06,7 \\
\hline & Negativo & 80 & 54,4 & 04 & 26,7 \\
\hline & Não realizado & 25 & 17,0 & 03 & 20,0 \\
\hline & Não preenchidas & 18 & 12,3 & 03 & 20,0 \\
\hline & Em andamento & 14 & 9,50 & 04 & 26,7 \\
\hline \multirow[t]{6}{*}{ Resolução do caso } & Cura & 100 & 68,0 & - & - \\
\hline & Abandono & 15 & 10,2 & - & - \\
\hline & Transferência & 09 & 6,10 & - & - \\
\hline & Mudança diagnóstica & 03 & 2,00 & - & - \\
\hline & Óbito & 17 & 11,6 & - & - \\
\hline & Não preenchidas & 03 & 2,00 & - & - \\
\hline \multirow[t]{6}{*}{ Região de moradia } & Norte & 34 & 23,1 & 03 & 20,0 \\
\hline & Sul & 29 & 19,7 & 05 & 33,3 \\
\hline & Leste & 21 & 14,3 & 03 & 20,0 \\
\hline & Oeste & 25 & 17,0 & 01 & 06,7 \\
\hline & Centro & 22 & 15,0 & 02 & 13,3 \\
\hline & Não preenchidas & 16 & 10,9 & 01 & 06,7 \\
\hline
\end{tabular}

A faixa etária mais acometida foi a dos indivíduos de 41 a 60 anos (35,4\%), seguidos pelos de 21 a 40 anos (32,7\%). Entre os pacientes que abandonaram o tratamento, 46,7\% estavam na faixa etária de 21 a 40 anos, ou seja, eram adultos jovens. Este abandono pode estar relacionado ao estilo de vida desta população, que normalmente faz uso de bebidas alcoólicas e possui horários irregulares para a alimentação, fatores que podem contribuir para a interrupção do tratamento.

Em relação à finalização dos casos, verificouse que 100 (68\%) finalizaram o tratamento para cura; $15(10,2 \%)$ pacientes abandonaram o tratamento; 17 (11,6\%) evoluíram para óbito; três (2,0\%) não fecharam o caso, em virtude de estarem em tratamento até a coleta dos dados; $9(6,1 \%)$ foram transferidos para outras cidades para concluir o tratamento e três (2,0\%) não confirmaram o diagnóstico.

De acordo com as metas internacionais estabelecidas pela Organização Mundial da Saúde e pactuadas pelo governo brasileiro, deve-se buscar a cura de $85 \%$ dos casos estimados de $\mathrm{TB}^{(5)}$. Este dado não condiz com o nosso estudo, já que apenas $68 \%$ dos pacientes evoluíram para a cura.

É preconizado que os programas de controle da TB tenham taxa de abandono de tratamento inferior a $5 \%{ }^{(8)}$. No Brasil, em 2003, a taxa média de abandono do tratamento situou-se em 12\%; no Estado de São Paulo foi de $10,3 \%$, sendo que existem enormes diferenças regionais, variando de 6,4\% em João Pessoa e 18,7\% em Porto Alegre, respectivamente ${ }^{(9)}$. Em consequência desse abandono, estima-se que haverá o aumento do contágio, dos custos do tratamento, da morbimortalidade, e do surgimento de resistência às drogas.

Um estudo realizado em 1996, em Londrina, que teve por objetivo avaliar a efetividade do Programa de Controle de Tuberculose, verificou-se que 17,7\% entre 188 pacientes abandonaram o tratamento ${ }^{(10)}$. A redução na freqüência de pacientes com TB que abandonaram o tratamento pode estar relacionada à descentralização do atendimento, ocorrida em 2002.

O Plano Nacional de Controle da Tuberculose no Brasil, implantado pelo Ministério da Saúde em 
1998, descentralizou para o nível municipal a responsabilidade relacionada à implementação de algumas ações da atenção básica à saúde, incluindo a atenção à TB desde a prevenção, diagnóstico, tratamento e acompanhamento dos pacientes, contribuindo, deste modo, para a expansão das ações de controle desta doença. Este Plano visa, junto com a atuação das Equipes de Saúde da Família, melhorar a adesão terapêutica e evitar o abandono do tratamento ${ }^{(11)}$.

Observou-se, em relação ao abandono do tratamento, na cidade de Bucaramanga, Colômbia, no ano 2007, que de 261 prontuários de pacientes com TB analisados, $14,9 \%$ registraram abandono de tratamento e, destes casos, 24,54\% dos pacientes tinham entre 21 a 40 anos. Dos 261 pacientes, 62,1\% eram do sexo masculino. Entre os fatores de risco considerados pelos autores ligados ao abandono do tratamento estavam o baixo nível socioeconômico, ser do sexo masculino e ter entre 21 e 30 anos $^{(12)}$.

Com relação ao tipo de entrada para o tratamento e o tipo de TB, o estudo mostra que $95,3 \%$ dos pacientes eram casos novos, $69,4 \%$ tinham como diagnóstico de entrada tuberculose pulmonar e 24,5\% tinham o diagnóstico de entrada tuberculose extrapulmonar. O mesmo pode ser observado com os pacientes que abandonaram o tratamento, pois $86,7 \%$ eram casos novos, $66,7 \%$ tinham como diagnóstico de entrada tuberculose pulmonar e 33,3\% tinham como diagnóstico de entrada tuberculose extrapulmonar. Conforme a Organização Mundial de Saúde, cerca de $90 \%$ dos casos existentes são de forma pulmonar da doença, enquanto as formas extrapulmonares correspondem a cerca de $10 \%{ }^{(6)}$.

Em relação aos agravos associados, observouse que $62,6 \%$ tinham o registro “ignorado”. Quando preenchido, observou-se que a maioria dos casos tinha o alcoolismo como agravo, representando $13,6 \%$ do total de pacientes e 13,3\% dos que não concluíram o tratamento. Na confirmação da sorologia para o vírus causador da aids, 54,4\% tiveram resultado negativo, $6,8 \%$ positivo, $17 \%$ não realizaram o exame, $12,3 \%$ das fichas não estavam preenchidas neste campo e $9,5 \%$ dos exames estavam em andamento. Do total de pacientes que abandonaram o tratamento, 26,7\% tinham sorologia negativa e 6,7\% tinham exame positivo. Já nos casos de abandono do tratamento, 73,3\% dos campos de agravos associados estavam como "ignorado" ou não preenchidos. No caso da realização do exame para HIV, dos pacientes que abandonaram o tratamento, 20\% estavam com este campo não preenchido e 20\% como não realizado.

A associação entre o HIV e o bacilo de Koch constitui um sério problema de saúde pública podendo levar ao aumento da morbimortalidade em muitos países, pois um paciente infectado com o vírus do HIV tem $45 \%$ mais chance de contrair o Mycobacterium tuberculosis ${ }^{(7)}$.

Outros fatores relacionados ao abandono do tratamento como baixa escolaridade, não-estruturação familiar, baixo número de componentes na família, nãoinserção no mercado de trabalho e ausência de vínculo empregatício foram encontrados em um estudo semelhante realizado no Município do Rio de Janeiro, em que os fatores acima apresentaram dados estatisticamente significativos ${ }^{(13)}$.

Tendo como base a região de moradia dos pacientes para uma avaliação socioeconômica, pudemos observar que 23,1\% moravam na região Norte e 19,7\% na região Sul de Londrina, entre os pacientes que não concluíram o tratamento, 33,3\% residiam na região Sul. Essa região foi dividida em Sul A e B para melhor atendimento à população no que tange à atenção à saúde e assistência social. A região Sul A possui áreas de ocupação irregular, assentamentos e favelas que estão se tornando novos bairros, com baixo índice socioeconômico, condições de moradia precárias, sem infraestrutura e rede de esgoto. Nesta, houve maior concentração de pessoas que abandonaram o tratamento de TB. Na região Sul B, considerada uma área nobre da cidade, não houve caso de TB notificado.

As taxas de incidência mais elevadas do agravo em Londrina, em 2005, foram observadas no Jardim União da Vitória (região Sul), com 134 casos/100.000 habitantes; na Vila Fraternidade (região Sul), com 87/ 100.000 habitantes e no Jardim Itapoã, com 99/100.000 habitantes (região Norte) ${ }^{(2)}$.

Fatores relacionados à pobreza, como a baixa escolaridade e região de moradia, apresentados neste trabalho e apontados em outros estudos ${ }^{(7,13-14)}$, enfatizam o teor social da doença. Entretanto, é importante lembrar que além dos fatores ligados ao paciente, os relacionados ao serviço também são relevantes, como dificuldades nos aspectos organizacionais, entraves burocráticos durante o início do tratamento, forma de abordagem pela equipe, capacitação dos profissionais, entre outros $^{(14)}$.

Um estudo realizado em 2007, em João Pessoa, Paraíba, investigou a história de abandono de tratamento de TB e mostrou que $75 \%$ dos pacientes eram do sexo masculino e a totalidade tinha baixa esco- 
laridade, entre 6 a 8 anos de estudo ${ }^{(9)}$. Estes dados são semelhantes aos encontrados em Londrina, onde $80 \%$ dos pacientes que abandonaram o tratamento eram do sexo masculino, e 33,3\% do total de abandonos possuíam de quatro a sete anos de estudo.

Muitos estudos relatam que indivíduos com problemas concomitantes ao tratamento, como o etilismo, por exemplo, apresentam maior dificuldade na obtenção do sucesso terapêutico ${ }^{(7,12,14)}$. A falta de preenchimento do campo de agravos associados não possibilitou relacionar o alcoolismo com o abandono do tratamento, considerando que apenas $13,3 \%$ dos pacientes que abandonaram o tratamento tinham registro do agravo alcoolismo e que, em $73,3 \%$ desses, o campo de agravos da ficha de notificação não estava preenchido ou continha a anotação "ignorado".

Quanto à cor, a maior frequência dos que tiveram TB registrada na ficha SINAN foi de brancos (53,3\%), totalizando 33,3\% dos que abandonaram o tratamento. Estes dados podem estar relacionados ao perfil da população londrinense, onde a maioria $(74,2 \%)$ é branca $^{(15)}$, e não à predisposição racial para a TB. O que podemos observar é que, no mundo, a prevalência dessa doença entre negros chega a ser duas vezes maior do que em brancos. Porém, deve-se considerar que as taxas de prevalência mais elevadas são verificadas na África ${ }^{(16)}$. Isto nos leva a perceber que a relação deste dado com as condições socioeconômicas pode ser mais relevante do que a relação com a etnia. A falta de recursos para alimentação e locomoção adequadas, além do uso de álcool e outras drogas, merecem destaque nesse cenário. História de não-adesão anterior, efeitos adversos, não-aceitação do diagnóstico e melhora dos sintomas também têm importante papel no uso inconstante das medicações ${ }^{(17)}$.

Outro fator relacionado à redução das taxas de abandono do tratamento da tuberculose, não explicitado neste trabalho, porém de grande importância em vários estudos $^{(6,9,17)}$ é a atuação dos profissionais da Estratégia Saúde da Família, na qual é utilizada como paradigma a educação em saúde e o acolhimento dos pacientes com TB, considerando seus valores e crenças. É importante ressaltar que, algumas vezes, o serviço de saúde abandona o doente e negligencia o acompanhamento dos casos, fragilizando as relações imprescindíveis ao êxito do tratamento.

Conhecer o perfil dos pacientes com TB que abandonam o tratamento e capacitar os profissionais da saúde para o acolhimento ideal, humanizado, baseado na supervisão constante e no incentivo à adesão ao tratamento, contribuirá muito para a reversão das taxas de abandono na cidade de Londrina, aumentando os indicadores relacionados à cura desta patologia.

\section{CONCLUSÃO}

Em pleno século 21, muitos sofrem e morrem devido à TB, apesar de ser uma doença curável, do tratamento ser totalmente custeado pelo Sistema Único de Saúde, e de depender de exames simples e acessíveis. Este cenário tem relação com vários fatores que não se restringem apenas às políticas de saúde, envolvendo dimensões culturais, econômicas, sociais e ideológicas, motivo que dificulta o controle da doença. A identificação de pacientes com maior risco para abandono do tratamento justifica uma abordagem cuidadosa a ser definida conforme as condições próprias de cada local. Frente ao diagnóstico de TB, a adesão ao tratamento não só é importante para o estado de saúde dos doentes, mas constitui um problema de saúde pública.

Sendo assim, se faz necessária a criação de um modelo de assistência voltado ao perfil dos usuários que abandonam o tratamento, com uma prática participativa, coletiva, com grupos educativos que promovam ações preventivas, identificando e estudando estratégias para a superação de barreiras ligadas à realidade de vida, individual e coletiva.

Espera-se que os resultados desta pesquisa possam contribuir para a melhoria das ações de saúde em relação aos pacientes que tratam TB, fornecendo subsídios para as equipes intensificarem ações educativas e de conscientização sobre a necessidade de seguir o tratamento até a cura, diminuindo os casos de abandono do tratamento.

\section{REFERÊNCIAS}

1. Ferreira AAA, Queiroz KCS, Torres KP, Ferreira MAF, Accioly H, Alves MSCF. Os fatores associados à tuberculose pulmonar e à baciloscopia: uma contribuição ao diagnóstico nos serviços de saúde pública. Rev Bras Epidemiol. [Internet] 2005;8(2) [acesso em 12 dez 2008]. Disponível:http://www.scielosp.org/ pdf/rbepid/v8n2/06.pdf

2. Londrina. Secretaria Municipal de Saúde. Plano municipal de controle da tuberculose. Londrina; 2005. p. 6-9.

3. World Health Organization. Control mundial de la 
tuberculosis 2008 [acesso em 20 nov 2009] Disponível: http://www.who.int/tb/publications/global_report/2008/ key_points/es/index.html

4. Ministério da Saúde (BR). Fundação Nacional de Saúde. Centro de Referência Professor Hélio Fraga. Sociedade Brasileira de Pneumologia e Tisiologia. Controle da tuberculose: uma proposta de integração ensinoserviço. $5^{\mathrm{a}}$ ed. Rio de Janeiro: FUNASA; 2002.

5. Castelo FA, Kritski AL, Barreto AW, Lemos ACM, Netto AR, Guimarães CA, et al. II Consenso Brasileiro de Tuberculose: diretrizes brasileiras para tuberculose. J Bras Pneumol. [Internet] 2004;30(Supl1) [acesso em 10 nov 2008]. Disponível: http://www.scielo.br/pdf/ jbpneu/v30s1/a01bv30s1.pdf

6. Silveira MPT, Adorno RFR, Fontana T. Perfil dos pacientes com tuberculose e avaliação do Programa Nacional de Controle da Tuberculose em Bagé (RS). J Bras Pneumol. [Internet] 2007;33(2) [acesso em 15 nov 2008]. Disponível: http://www.scielo.br/pdf/jbpneu/v33 n2/12.pdf

7. Ribeiro SA, Amado VM, Fernandes MMA, Schenkman S. Estudo caso-controle de indicadores de abandono em doentes com tuberculose. J Bras Pneumol. [Internet] 2000;26(6) [acesso em 11 nov 2008]. Disponível: http:// www.jornaldepneumologia.com.br/pdf/2000_26_6_2_ portugues.pdf

8. World Health Organization. Global tuberculosis control: surveillance, planning, financing. 2005 [ acesso em 11 nov 2008]. Disponível: http://apps.who.int/gb/ebwha/ pdf_files/WHA58/A58_7-sp.pdf

9. Sá LD, Souza KMJ, Nunes MG, Palha PF, Nogueira JA, Villa TCS. Tratamento da tuberculose em unidades de saúde da família: histórias de abandono. Texto Contexto Enferm. [Internet] 2007;16(4) [acesso em $14 \mathrm{dez} 2008$ ]. Disponível: http://www.scielo.br/pdf/tce/v16n4/a16v16 n4.pdf

10. Melo VO, Soares DA, Andrade SM. Avaliação do programa de controle da tuberculose em Londrina - PR no ano 1996. Inf Epidemiol SUS. 1999;8(4):53-62.

11. Ministério da Saúde (BR). Secretaria de Políticas de Saúde. Departamento de Atenção Básica. Manual técnico para o controle da tuberculose: cadernos de atenção básica. Brasília; 2002.

12. Cáceres FM, Orosco LC. Incidencia y factores asociados al abandono del tratamiento antituberculoso. Rev Biomed. [Internet] 2007;27(6) [acesso em 12 dez 2008].
Disponivel: http://www.scielo.org.co/

13. Natal S, Valente J, Gerhadt G, Penna ML. Modelo de predição para o abandono do tratamento da tuberculose pulmonar. Bol Pneumol Sanit. [Internet] 1999;7(1) [acesso em 12 dez 2008]. Disponível: http://scielo.iec.pa. gov.br/pdf/bps/v7n1/v7n1a07.pdf

14. Brasil PMAA. Fatores predisponentes de abandono de tratamento de tuberculose: uma metanálise [tese]. Rio de Janeiro (RJ): Universidade Estadual do Rio de Janeiro; 2006.

15. Silva MN. O negro em Londrina: da presença pioneira negada à fragilidade das ações afirmativas na UEL. Rev Espaço Acad. [Internet] 2008;7(82) [acesso em 15 out 2008]. Disponível: http://www.espacoacademico.com.br/ 082/82silva.pdf

16. Mendes AM, Fensterseifer LM. Tuberculose: por que os pacientes abandonam o tratamento? Bol Pneumol Sanit. [Internet] 2004;12(1) [acesso em 11 nov 2008]. Disponível: http://scielo.iec.pa.gov.br/

17. Nuevo informe sobre la carga mundial de la tuberculosis y su relación com la infección por el VIH. Rev Panam Salud Publica. [Internet] 2003;14(1) [acesso em $11 \mathrm{dez}$ 2008]. Disponível: http://www.scielosp.org/pdf/rpsp/v 14n1/16634.pdf 